\title{
Aceros inoxidables de nuevo diseño resistentes a la corrosión localizada ${ }^{(\cdot)}$
}

\author{
E. Otero ${ }^{*}$, J. Botella ${ }^{* *}, J$. Botana ${ }^{* * *}$, V. Matres ${ }^{* *}$ y R. Merello** \\ Resumen Un nuevo material suele ser un material clásico al que pequeñas modificaciones \\ proporcionan nuevas y/o mejores propiedades, haciéndolo competitivo desde el punto de \\ vista del mercado. De acuerdo con esta filosofía, se describe en el presente trabajo el \\ desarrollo de un nuevo acero inoxidable austenítico sobre la base del AISI 304 modificado, \\ con propiedades de resistencia a la corrosión por picadura similares o, incluso, superiores a \\ un AISI 316, y el de un acero inoxidable dúplex, de bajo contenido en níquel y \\ comportamiento frente a la corrosión por picadura similar a un dúplex convencional. En \\ ambos casos, la relación prestaciones/precio resulta ser competitiva. El trabajo se inscribe en \\ el marco del plan general de I+D que desarrolla Acerinox S.A. en colaboración con varios \\ centros públicos de investigación en el área de la Ciencia y Tecnología de los Materiales.
}

Palabras clave Corrosión por picadura. Acero inoxidable.

\section{New design pitting corrosion resistance stainless steel}

\begin{abstract}
A new material, usually, is a classic material with small modifications. The modifications provide new and/or superior properties to the material, making it competitive. The development of a new austenitic stainless steel based on the modified AISI 304 is described in the present work. This new steel shows a pitting corrosion resistance similar, or even better, than AISI 316. The behaviour of a stainless duplex steel with a low content in $\mathrm{Ni}$ is also described here. Its pitting corrosion resistance is similar than a conventional duplex steel. In both cases the ratio properties/price turns out to be competitive. The work was carried out by Acerinox S.A. inside the general plan of $I+D$, which is developed in collaboration with several public research centers whose principal activities are related to Science and Technology of Materials.
\end{abstract}

Keywords Pitting corrosion. Stainless steel.

\section{INTRODUCCIÓN}

Lo que a continuación se expone, en el presente trabajo, constituye una parte de la investigación llevada a cabo, a lo largo de los últimos años, por el Centro de I+D de Acerinox S.A. en colaboración con Centros de Investigación en el área de Ciencia y Tecnología de Materiales de las Universidades de Cádiz, Sevilla y Complutense y Rey Juan Carlos de Madrid, encaminada al diseño de nuevos aceros inoxidables con propiedades mejoradas y precio competitivo.

En concreto, se abordan los trabajos desarrollados en recientes Tesis Doctorales de la Universidad Complutense de Madrid ${ }^{[1]}$ y de la Universidad de Cádiz ${ }^{[2]}$.

El objetivo de estos trabajos es, respectivamente, el desarrollo de un acero inoxidable austenítico del tipo AISI 304 modificado, con propiedades de resistencia a la corrosión por picadura, similar a un AISI 316 y el desarrollo de un acero inoxidable dúplex con bajo contenido en níquel con propiedades de resistencia a la corrosión por picadura similar a un dúplex convencional. En ambos casos, el material tendría un precio competitivo.

\section{ACERO INOXIDABLE AUSTENÍTICO TIPO AISI 304, MODIFICADO CON RESISTENCIA A LA CORROSIÓN POR PICADURA, SIMILAR AL AISI 316}

\subsection{Aceros inoxidables austeníticos}

Son aleaciones $\mathrm{Fe}-\mathrm{Cr}$ en las que el níquel entra como uno de los elementos mayoritarios

\footnotetext{
(•) Trabajo recibido el día 6 de noviembre de 2003 y aceptado en su forma final el día 24 de febrero de 2005.

$\left(^{*} \quad\right.$ Universidad Rey Juan Carlos. C/ Tulipán, s/n. 28933 Móstoles, Madrid (Spain).

${ }^{* *}$ ACERINOX. Apartado 83 Palmones, Los Barrios, 11370 Algeciras, Cádiz (España).

$\left(^{* * *}\right)$ Universidad de Cádiz, Dpto. de Ciencia de Materiales e Ingeniería Metalúrgica, Pol. Río San Pedro, s/n. Apartado $40 ; 11510$ Puerto Real, Cádiz, (España).
} 
(generalmente, por encima del $8 \%$ ); de cualquier manera, su composición es equilibrada, con el fin de que, a la temperatura ordinaria, bien sea en estado estable o metaestable, su estructura sea totalmente austenítica. La adición de molibdeno mejora su resistencia a la corrosión por picadura en medios ácidos con cloruros y a la corrosión bajo tensión.

El acero inoxidable austenítico más característico, con resistencia a la corrosión por picadura en soluciones cloruradas es el AISI 316 (17Cr11Ni2Mo). El elemento químico diferenciador con respecto al AISI 304 (18Cr8Ni), con vistas al comportamiento a la corrosión por picaduras, es el molibdeno. Las diferencias en los contenidos de níquel de ambas aleaciones no son relevantes en cuanto a su influencia en la resistencia a la corrosión localizada y, el mayor nivel de níquel del 316 sirve para restablecer el balance austenítico de la aleación, compensando el fuerte efecto alfágeno del molibdeno. Para establecer el comportamiento a la corrosión por picadura en las aleaciones clásicas (AISI 316, 3161, 317, etc..) se emplea el llamado Pitting Resistance Equivalent (PRE), definido como:

$$
\mathrm{PRE}=\mathrm{Cr}+3,3 \mathrm{Mo}+\mathrm{nN}
$$

donde cromo, molibdeno y nitrógeno se expresan como porcentaje en masa y donde, según diversos autores $^{[3-7]}$, el coeficiente que afecta al nitrógeno puede oscilar entre $\mathrm{n}=12,8$ y $\mathrm{n}=30$.

La ecuación (1) pone claramente de manifiesto que la resistencia a la corrosión por picadura es un fenómeno que tiene que ver con la acción sinérgica de los aleantes, cromo, molibdeno y nitrógeno.

En los últimos años, los nuevos diseños de inoxidables tienen en cuenta al nitrógeno como aleante y no sólo desde el punto de vista de la acción sinérgica, para la resistencia a la corrosión localizada. El nitrógeno actúa como un elemento fuertemente gammágeno y como gran estabilizador de la austenita frente a las transformaciones martensíticas (por tratamiento o por deformación). La aleación con nitrógeno permite, también, obtener importantes efectos en la elevación de las propieda- des mecánicas.

De acuerdo con lo anterior, el empleo de nitrógeno permite una parcial sustitución del níquel como aleante gammágeno, pero la aleación con nitrógeno se ve limitada -en los procesos metalúrgicos normales de las Acerías- por su relativa baja solubilidad en el acero y por sus abruptos cambios de solubilidad durante la solidificación del metal. Uno de los recursos metalúrgicos para alear con nitrógeno, salvando las dificultades derivadas de su solubilidad, consiste en disminuir el aporte a la aleación y aumentar el de manganeso.

En este sentido, fueron desarrollados los aceros inoxidables de la serie 200 de AISI, intentando afrontar la crisis del mercado del níquel derivada de la última guerra mundial. El típico representante de estos aceros, $17 \mathrm{Cr} 5 \mathrm{Ni} 6,5 \mathrm{Mo} 0,15 \mathrm{~N}$, intentaba sustituir al $18 \mathrm{Cr} 8 \mathrm{Ni1}, 5 \mathrm{Mn}$ (AISI 304), ahorrando un $3 \%$ de níquel de aleación. Con respecto al AISI 316 existen, también, algunas fórmulas de la misma época que, mediante el empleo de nitrógeno,manganeso y molibdeno, ahorraban níquel también en aleaciones sustitutivas. Sin embargo, aquellos aceros de la serie 200 no prosperaron, debido a una cierta estabilidad posterior del mercado del níquel, a problemas de fabricación en las acerías, por la erosión que provocaba en los refractarios la alta concentración de manganeso, así como el endurecimiento estructural provocado por el nitrógeno en los aceros, afectando a la deformabilidad en frío y, finalmente, al peor comportamiento a la corrosión (p. ej.: por picaduras) ${ }^{[8-10]}$.

Una de las principales causas del inicio y desarrollo de las picaduras en medios clorurados es la presencia de inclusiones tipo $\mathrm{MnS}^{[11-15]}$ como la mostrada en la figura 1.

De acuerdo con lo anterior, parece razonable postular que, en la medida en la que en un determinado tipo de acero se redujese la presencia de inclusiones tipo $\mathrm{MnS}$ mejoraría el comportamiento a la corrosión por picaduras. La mejor opción, en este sentido, sería eliminar totalmente la

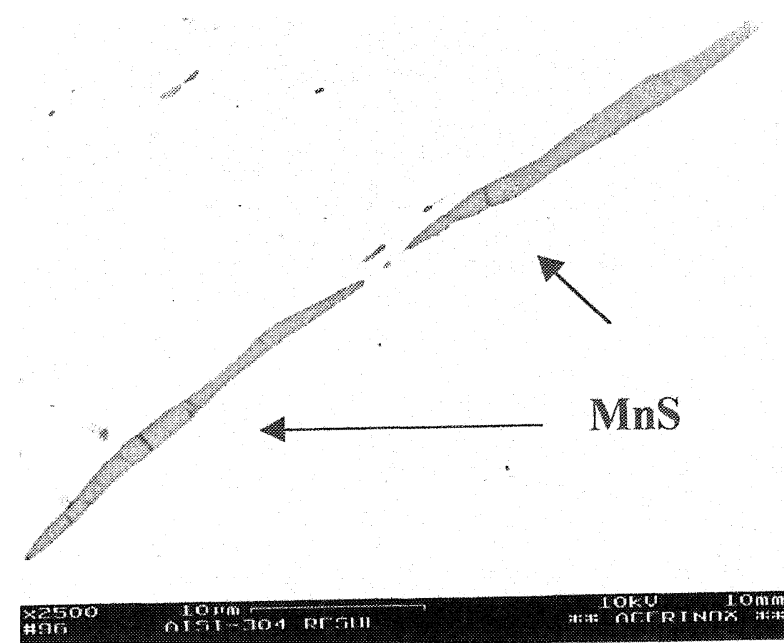

Figura 1. Imagen de electrones retrodispersados de una sección paralela de laminación de un acero inoxidable austenítico AISI 304, mostrando inclusiones de MnS.

Figure 1. Backscattered electron image showing MnS inclusions in the section of an austenitic stainless steel AISI 304. 
presencia de sulfuros de manganeso, lo que, para un cierto nivel de manganeso, haría necesario disminuir los contenidos de azufre por debajo de su límite de solubilidad en el acero a la temperatura ambiente, que se sitúa alrededor de $10 \mathrm{ppm}$.

De acuerdo con los antecedentes, empresas productoras como Acerinox y Centros Universitarios como las Universidades de Cádiz, Sevilla o Complutense y Rey Juan Carlos de Madrid, trabajan en el desarrollo de una serie de materiales nuevos, recogiendo aportaciones previas recientes ${ }^{[16-18]}$, algunas de ellas desarrolladas en Programas Europeos altamente competitivos.

\subsection{Diseño de un 304 modificado, resistente a la corrosión por picadura}

Un nuevo material puede y suele ser un material clásico, al que pequeñas modificaciones confieren nuevas y mejores propiedades. En el caso de esta propuesta se estudian las sinergias positivas de los aleantes clásicos (para reforzarlas) y las negativas (para eliminarlas), de modo que se consigan resultados solventes transferibles tecnológicamente.

De acuerdo con lo expuesto, se deduce que es posible apoyarse en varias sinergias para conseguir diseñar aleaciones tipo AISI 304, con similar resistencia a la corrosión por picaduras que la del tipo AISI 316, que se basan en lo siguiente:

a) Una aleación con la composición química del AISI 304 pero con el azufre por debajo del límite de solubilidad $(\mathrm{S}<10 \mathrm{ppm})$.

b) Además, debe tener $\mathrm{Mn}<0,2 \%$ para conseguir la sinergia, pues, así, se aumenta la solubilidad del azufre, evitando aún más la formación de sulfuros del tipo $\mathrm{MnS}$, puesto que, en estas condiciones, si se formase algún sulfuro, sería del tipo $\mathrm{CryS} z$ (resistente a la corrosión) y no del tipo MnS (fácilmente corroíble).

c) Fabricar la aleación 304LSMn (es decir $\mathrm{S}<10$ ppm, $M n<0,2 \%$ ) aleando con nitrógeno: si los contenidos habituales del nitrógeno en el AISI 304 suelen ser de 500 ppm, la propuesta se basa en alear este nuevo tipo de 304 con nitrógeno próximo a las $1.000 \mathrm{ppm}$. Con ello, se conseguiría compensar la pérdida del posible efecto gammágeno del manganeso y de su seguro efecto de estabilidad de la austenita, debido a la reducción de su concentración habitual. Por otra parte, el nitrógeno daría al acero mejores propiedades mecánicas y actuaría sinérgicamente con cromo y molibdeno en la mejora de la re- sistencia a la corrosión por picaduras, reforzando las acciones a) y b).

d) Alear con un nivel de molibdeno próximo al típico, que se obtiene habitualmente en la fabricación, como molibdeno residual procedente de la fusión de las chatarras, de los AISI 304 comerciales. Dicho nivel de molibdeno igual a 0,2-0,4\% aseguraría el efecto sinérgico de cromo, molibdeno y nitrógeno difícil de darse si falta alguno de dichos elementos en la composición del acero.

Tal esquema, también es trasladable al AISI 316, para poder conseguir comportamientos mejorados de esta aleación a la corrosión por picaduras y comparables al AISI 317.

\subsection{Correlación entre los valores de Er y el con- tenido en azufre de las aleaciones tipo 304 y 316}

Se han establecido, experimentalmente, las relaciones entre los contenidos en azufre y el potencial de picadura para aceros tipo 304 y 316. Las relaciones exponenciales que ligan estas variables y la representación gráfica de los resultados experimentales obtenidos se muestran en las figuras 2 y 3 .

Parece claro que el porcentaje de azufre en la composición química de la aleación influye significativamente en la resistencia a la corrosión por picaduras, de forma que, al elevarse el contenido en azufre disminuye el potencial de picaduras. Esto, es cierto hasta determinados valores de azufre, a partir de los cuales, un incremento del contenido de azufre no supone una apreciable disminución adicional del potencial de picadura. Este valor se sitúa en $0,0043 \%$ en masa.

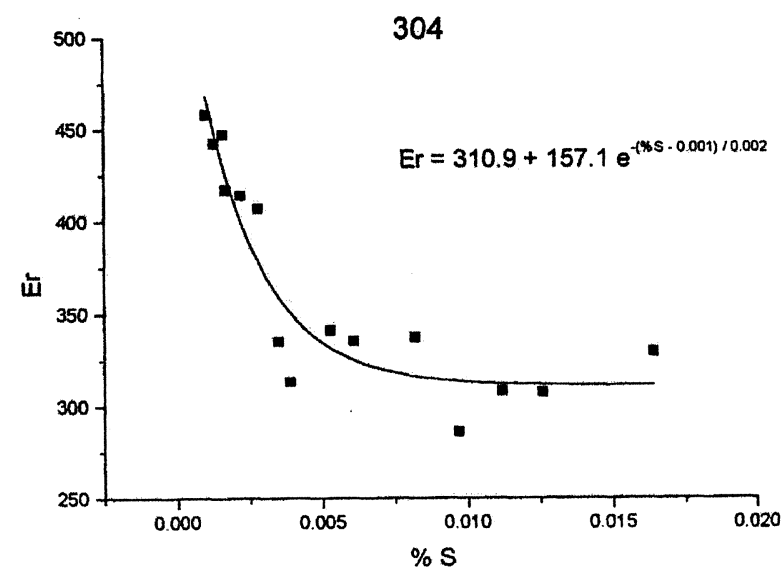

Figura 2. Er frente al \% de azufre. Tipo 304.

Figure 2. Er Vs. \% S. 304 Type. 


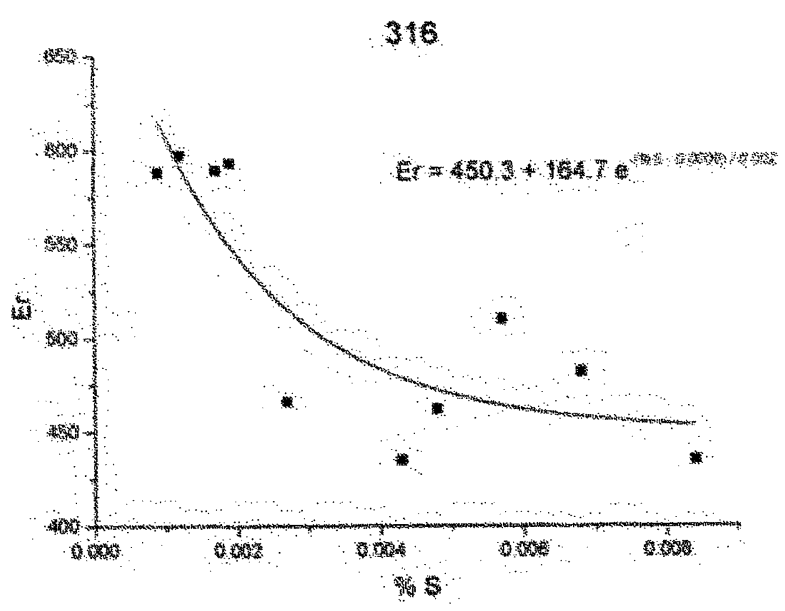

Figura 3. Er frente al \% de azufre. Tipo 316.

Figure 3. Er Vs. \% S. 316 Type.

A partir de lo anterior se puede establecer lo siguiente:

a) Un porcentaje de $\mathrm{S}<0,0010$ (10 ppm) no afecta negativamente al potencial de picadura de la aleación, que alcanzaría para estos valores, de acuerdo con la relación exponencial de la figura 2, el máximo:

$$
\mathrm{Er}_{\max }=310,9+157,1=468 \mathrm{mV}
$$

b) El potencial de rotura del AISI304 disminuye rápidamente $\mathrm{y}$, en consecuencia, la resistencia a la corrosión por picadura del material, conforme se incrementa el contenido de azufre del mismo, entre determinados límites:

$$
0,0043>\mathrm{S}>0,0010
$$

Por encima del valor $S=0,0043$ y hasta $S=$ 0,0164 , no se producen, prácticamente, caídas adicionales de los potenciales de picadura, lo que parece indicar que, a partir del nivel de azufre $S=$ 0,0043 , el control de potencial de picadura dejaría de depender de la cantidad de azufre y pasaría a depender de otros factores, como, por ejemplo, del resto de la composición química en cromo, molibdeno y nitrógeno como elementos determinantes.

El resultado obtenido para el AISI 316, de acuerdo con la ecuación exponencial descrita en la figura 3, es similar: 0,0043 \% en masa.

A partir de este resultado se puede establecer que:

a) Un porcentaje de $\mathrm{S}=0,0009$ (9 $\mathrm{ppm}$ ) no afecta negativamente al potencial de picadura de la aleación, que alcanzaría para éste valor, el máximo:

$$
\mathrm{Er}_{\text {max }}=450,3+164,7=615 \mathrm{mV}
$$

b) El porcentaje de rotura por picadura, del AISI 316 , se reduce rápidamente y, por tanto, la resistencia del material a la corrosión por picadura, conforme se incremente el contenido de azufre entre determinados límites:

$$
0,0043>S>0,0009
$$

Por encima del valor $S=0,0043$ y hasta $S=$ 0,0084 no se producen, prácticamente, caídas del potencial de picadura, lo que parece indicar que, al igual que ocurría con los aceros inoxidables tipo 304 , a partir del nivel de $S=0,0043$, dejaría de tener el control del potencial de picadura la cantidad de azufre (consecuentemente la cantidad de sulfuros) y pasaría a depender de otros factores derivados, fundamentalmente, del resto de la composición del acero.

\subsection{Correlación entre los valores de Er y el PRE en aleaciones tipo 304 y 316}

Se ha establecido, también, una correlación entre los valores de potencial de rotura (Er) obtenidos para las aleaciones 304 y 316 y las variables de la composición química que se consideran influyentes en la resistencia a la corrosión por picadura.

Parece razonable partir, como una de las variables de composición química que influyen en el potencial de rotura, de la expresión bien establecida del $\mathrm{PRE}=\% \mathrm{Cr}+3,3 \% \mathrm{Mo}+30 \% \mathrm{~N}$, ya que la otra variable (el azufre), a partir de determinados valores, resulta independiente, en su efecto negativo o, como hemos establecido, de que la aleación sea tipo 304 o tipo 316. Dicha correlación es la siguiente:

$\mathrm{Er}=-364,2+144,2 \mathrm{e}^{-(\% \mathrm{~S}-0,001) / 0,002}+32,1$ PRE

La figura 4 presenta el ajuste de potenciales teóricamente obtenidos mediante dicha ecuación y los potenciales experimentales, observándose una buena aproximación lo que concede, sin duda, a la citada ecuación un gran interés tecnológico, puesto que permite predecir el comportamiento frente a la corrosión por picadura a partir de los valores de composición de un acero inoxidable austenítico perteneciente a las familias de 304 y 316. 
$304+316$

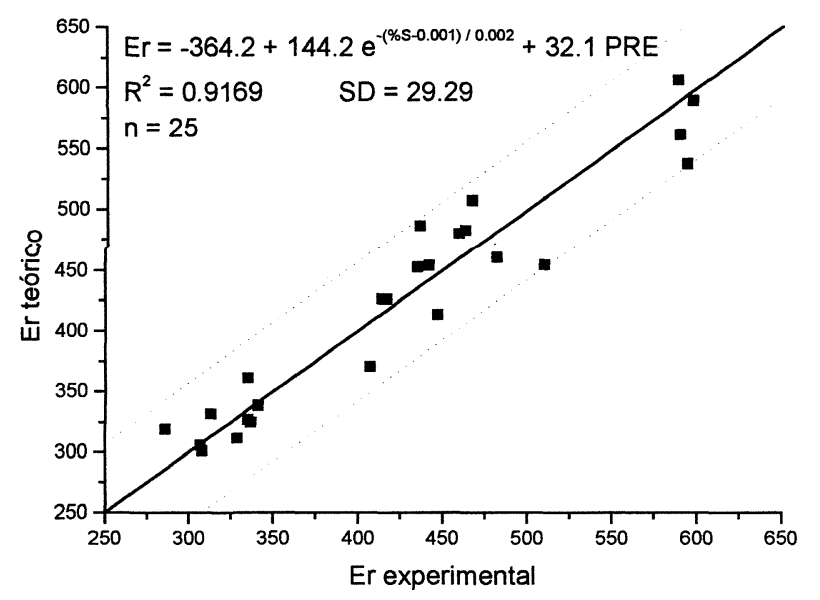

Figura 4. Er teórico frente a Er experimental. 304+316.

Figure 4. Theoretical Er Vs. Experimental Er. 304+316.

\subsection{Diseño de una nueva aleación resistente a la corrosión por picadura}

Teniendo en cuenta todo lo dicho anteriormente, se puede diseñar un nuevo material, introduciendo pequeñas variaciones en la composición química de un determinado acero inoxidable que se considere como base de partida experimental de modo que, el acero modificado presente sustanciales mejoras con respecto al acero base, previstas de antemano en cuanto al comportamiento a la corrosión por picadura.

La premisa de esta aleación es la poseer un nivel de azufre lo más cercano posible al límite de su solubilidad en el acero, de forma que desaparezca la posibilidad de formación de inclusiones del tipo de los sulfuros de manganeso. Se establece, por tanto, un valor deseado de $\mathrm{S}=0,001 \%$, de acuerdo con los resultados obtenidos, en los que se han puesto de manifiesto límites de solubilidad del orden de 10 ppm de azufre en el AISI 304. Por otro lado, se propone un ligero aumento del molibdeno, hasta el $0,4 \%$, ya que es un elemento que mejora la resistencia de la aleación a la corrosión por picadura. Además, esta composición está en el entorno de la correspondiente a la chatarra utilizada en el proceso de obtención industrial. En la preparación del mismo, interesa que el manganeso no sea alto, como se ha comentado anteriormente, con el fin de que si se formara alguna inclusión de sulfuro, ésta fuera preferentemente de cromo, menos dañina, en la resistencia a la corrosión por picadura, que la del manganeso. En consecuencia, se rebajaría el contenido de manganeso hasta el 0,2\%, a la vez que se dejaría prácticamente sin cambios el nivel de cromo.

Puesto que el incremento de nitrógeno llevará consigo un endurecimiento estructural de la red cristalina, debido al efecto de la disolución en la austenita de relativamente altos niveles de este elemento intersticial y, puesto que también actuaría en dicho sentido el relativo incremento del elemento sustitucional molibdeno, la propuesta para el diseño debería contemplar la rebaja de carbono (también intersticial), a niveles del 0,020\%. Conservando los niveles de silicio y de cobre en los típicos del 304 clásico, tendríamos la composición química deseada (a excepción del nitrógeno) que figura en la tabla I.

Para esta aleación se calcula el porcentaje de nitógeno necesario, teniendo en cuenta que la presencia de ferrita d de solidificación no debe exceder del $5 \%$, con el fin de evitar problemas de fisuración durante el proceso de colada.

De acuerdo con lo anterior, los niveles de nitrógeno deberían ser de unas 1.200 ppm. Aplicando la ecuación (3) se obtendría para este nuevo material un potencial de $532 \mathrm{mV}$, que es un potencial más próximo (incluso superior) a los típicos del AISI 316 que a los del 304. Por tanto, la composición del nuevo material, en lo que se refiere a elementos influyentes en $\mathrm{Er}$, sería la ofrecida en la tabla II.

Los resultados experimentales obtenidos respecto al potencial de picadura para este acero resultaron ser de $\mathrm{Er}=530 \mathrm{mV}$, en excelente concordancia con lo previsto. Se trata, por tanto, de un nuevo material que podría considerarse un 304 modificado, con prestaciones iguales o superiores a un 316 , en cuanto a su comportamiento a la corrosión por picadura.

Tabla I. Composición química deseada de la nueva aleación Table I. New alloy's composition

\begin{tabular}{lcccccccc}
\hline \multicolumn{10}{c}{ \% en masa } \\
\hline $\mathrm{C}$ & $\mathrm{Cr}$ & $\mathrm{Ni}$ & $\mathrm{Mo}$ & $\mathrm{Cu}$ & $\mathrm{N}$ & $\mathrm{Mn}$ & $\mathrm{Si}$ & $\mathrm{S}$ \\
\hline 0,020 & 18,50 & 8,30 & 0.4 & 0,25 & $?$ & 0,2 & 0,3 & 0,001 \\
\hline
\end{tabular}

Tabla II. Elementos influyentes en $\mathrm{Er}$

Table II. Influential elements in $\mathrm{Er}$

\begin{tabular}{cccc}
\hline$\% \mathrm{Cr}$ & $\% \mathrm{Mo}$ & $\% \mathrm{~N}$ & $\% \mathrm{~S}$ \\
\hline 18,50 & 0,40 & 0,1200 & 0,0010 \\
\hline
\end{tabular}

Rev. Metal. Madrid 41 (2005) 148-158 
3. ACERO INOXIDABLE DÚPLEX BAJO EN NÍQUEL, CON RESISTENCIA A LA CORROSION POR PICADURA SIMILAR A UN DÚPLEX CONVENCIONAL

\subsection{Aceros inoxidables dúplex}

Los aceros inoxidables dúplex se caracterizan, fundamentalmente, por la presencia de una estructura bifásica compuesta por granos de ferrita $(\alpha)$ y austenita $(\gamma)$. La fracción volumétrica de cada fase depende, principalmente, de la composición química de la aleación y la temperatura de solubilización empleada. Los aceros dúplex están constituidos por cuatro elementos de aleación principales $(\mathrm{Cr}, \mathrm{Ni}$, Mo, N) y, en algunos casos, por otros aleantes secundarios $(\mathrm{Si}, \mathrm{Cu}, \mathrm{W}, \mathrm{Mn})$. Una de las formas más simplificadas de representar el diagrama de fases de estas aleaciones es a través del diagrama pseudobinario $\mathrm{Fe}-\mathrm{Cr}-\mathrm{Ni}$ que se muestra en la figura 5 , donde los contenidos de cromo y níquel de los aceros inoxidables dúplex han sido reemplazados por las relaciones $\mathrm{Cr}_{\text {equiv }} / \mathrm{Ni}_{\text {equiv }}$ correspondientes. La región sombreada indica el rango de composiciones de los aceros inoxidables comerciales y, en ella, se pueden apreciar, también, las relaciones $\mathrm{Cr}_{\text {equiv }} /$ $\mathrm{Ni}_{\text {equiv }}$ de tres aceros inoxidables dúplex conocidos comercialmente.

El comportamiento frente a la corrosión de los aceros dúplex es, sin lugar a duda, la característica más importante de estos materiales. Su resistencia

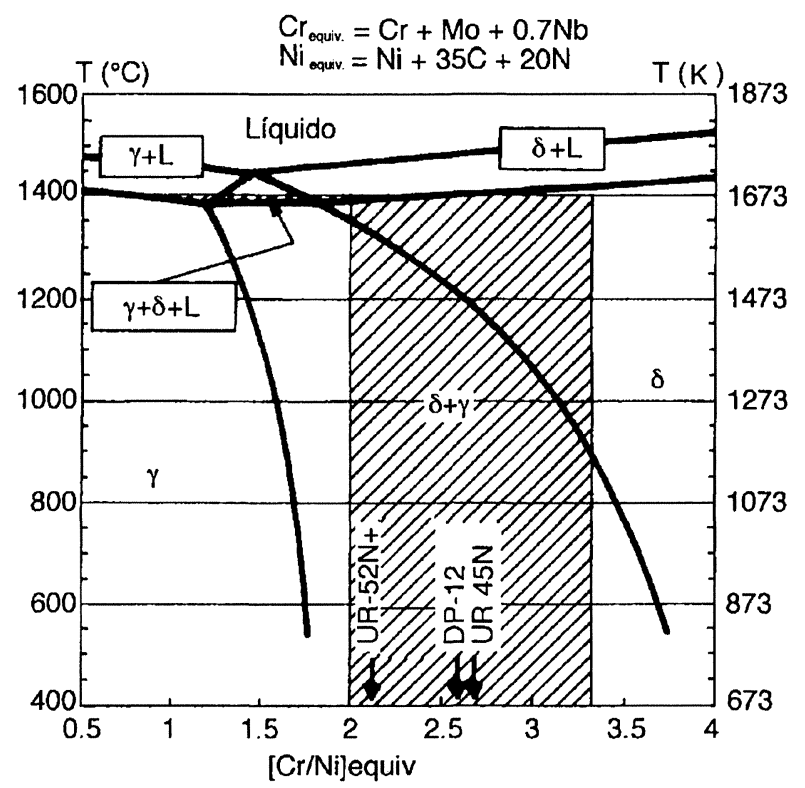

Figura 5. Diagrama pseudobinario $\mathrm{Fe}-\mathrm{Cr}-\mathrm{Ni}$.

Figure 5. Fe-Cr-Ni pseudobinary phase diagram. a muchos medios agresivos es comparable e, incluso, superior a la de los aceros inoxidables convencionales.

Los aceros inoxidables dúplex son, prácticamente, inmunes a la corrosión intergranular, debido a la precipitación de carburos de cromo del tipo $\mathrm{M}_{23} \mathrm{C}_{6}$. Esto, se debe a dos razones: la precipitación de carburos se producirá, preferencialmente, en la ferrita debido a su mayor contenido de cromo, produciendo un empobrecimiento de este elemento en las zonas adyacentes al precipitado. Sin embargo, debido a la mayor velocidad de difusión de los elementos de aleación de esta fase, el perfil de concentración de cromo en la región empobrecida resulta más ancho y menos profundo, reduciendo, con ello, el riesgo de sensibilización. Asimismo, el contenido de carbono tan bajo $(0,015 \%)$, que presentan los aceros inoxidables dúplex de última generación, inhibe, prácticamente, la precipitación de carburos de estos materiales.

La resistencia a la corrosión por picadura, en presencia de cloruros y otros aniones agresivos, es, en general, superior a la de los aceros inoxidables austeníticos. No obstante, la presencia de fases secundarias reduce de manera relevante la resistencia a la corrosión por picaduras de los aceros inoxidables dúplex, pues su presencia facilita el ataque localizado por disolución selectiva de las mismas (suelen presentar comportamiento anódico respecto a las fases mayoritarias) y reducen la estabilidad local de las películas pasivas.

Por último, es preciso destacar el buen comportamiento de estas aleaciones respecto a fenómenos de corrosión bajo tensión en presencia de cloruros, lo que les hace el sustituto adecuado, en muchas aplicaciones, de los aceros inoxidables austeníticos.

\subsection{Diseño de aceros inoxidables dúplex bajos en níquel, con buen comportamiento a la corrosión por picadura}

Los nuevos aceros inoxidables dúplex, a los que nos referiremos a continuación, tienen como diferencia fundamental con los dúplex clásicos, un menor contenido de níquel $(\leq 2,5 \%)$. Para compensar la disminución de un elemento como el níquel, esencialmente gammágeno, se eleva el nivel de nitrógeno, elemento a la vez fuertemente gammágeno y estabilizador de la austenita. Por otra parte, el contenido de manganeso se incrementa a valores de, aproximadamente, el $8 \%$, para favorecer la solubilidad del nitrógeno. El contenido de cobre se aumenta respecto a los contenidos habituales de este 
Aceros inoxidables de nuevo diseño resistentes a la corrosión localizada E. Otero, J. Botella, J. Botana, V. Matres y R. Merello

elemento en los dúplex clásicos, a un valor de, aproximadamente, un $1 \%$. De esta forma, el cobre se emplea en el diseño de los nuevos dúplex para reemplazar parte del efecto perdido por la disminución de níquel pero, también, para mejorar la resistencia a la corrosión del acero en determinados medios. Este diseño permite obtener aceros dúplex con un menor coste económico, gracias a la disminución del contenido de níquel y a la utilización de nitrógeno como aleante fundamental.

Los nuevos aceros inoxidables dúplex se han diseñado para que la relación de fases después de un tratamiento de homogeneización de $1.100{ }^{\circ} \mathrm{C}$, durante $30 \mathrm{~min}$, sea de $40-60 \%$ (en volumen). El aumento del contenido en nitrógeno, como aleante, tiene dificultades prácticas derivadas de la baja solubilidad en la austenita y casi nula en la ferri$\mathrm{ta}^{[19}{ }^{19}{ }^{24]}$. Por otra parte, aumenta la probabilidad de precipitación de nitruros como el de cromo. Para mejorar la solubilidad del nitrógeno se incrementan los niveles de cromo ${ }^{[26}$ y 27$]$ y, muy especialmente, los de manganeso ${ }^{[20 \text { y } 26]}$. La disminución de un elemento como el níquel, esencialmente gammágeno, se compensa, como ya se ha comentado, con el aumento del contenido de nitrógeno, con marcado carácter formador y estabilizador de la austenita ${ }^{[27]}$. El manganeso no va a intervenir, prácticamente, en el reparto de fases $\alpha / \gamma$, dado su papel ambiguo -ni alfágeno, ni gammágeno- a concentraciones relativamente altas ${ }^{[28 \text { y 29] }}$. No obstante, es claro su doble papel, fuertemente estabilizador de la austenita, por un lado, y de importante solubilizador del nitrógeno en el acero, por otro. Los contenidos de cromo se han mantenido equivalentes a los dúplex clásicos 2205, huyendo de niveles más altos por la tendencia del cromo a estimular la formación de fases. Este elemento, además de aumentar la solubilidad del nitrógeno, favorece la resistencia a la corrosión por picaduras ${ }^{[23}$ y 30$]$.

Respecto a otros elementos minoritarios, el azufre se mantiene en niveles inferiores a 0,002 \%. Aunque el contenido de manganeso es del $8 \%$, es poco probable la formación de MnS, al ser tan bajo el contenido de azufre y considerar la más alta solubilidad de dicho elemento en la fase ferrítica. Por otra parte, algunos autores argumentan que el efecto negativo del manganeso asociado a la formación de inclusiones de $\mathrm{MnS}$, se equilibra en los aceros con alto contenido en nitrógeno, ya que el manganeso aumenta la solubilidad del nitrógeno y, por tanto, su efecto beneficioso en la resistencia a la corrosión por picaduras $^{[31-33]}$. Por último, el fósforo se ha mantenido en niveles residuales en las nuevas aleaciones.

\subsection{Correlación entre Er y el PREN en aleacio- nes dúpex bajas en níquel}

A través de experimentación, se ha logrado relacionar los valores de potenciales de picadura con las composiciones químicas de las distintas aleaciones dúplex bajas en níquel preparadas y, más específicamente, con la composición de cada una de las fases a y g, en concreto, con los contenidos de los elementos fundamentales en la mejora de la resistencia a la corrosión por picaduras. Esta relación viene dada por la ecuación:

$$
E p=\exp (1,08+0,14 \text { PREN })
$$

en la que PREN viene definido por la ecuación:

$$
\mathrm{PREN}=\% \mathrm{Cr}+3,3 \% \mathrm{Mo}+16 \% \mathrm{~N}
$$

Esta expresión permite diseñar aleaciones dúplex, con bajos contenidos de níquel, con resistencias a la corrosión por picaduras equivalentes a las de los aceros dúplex clásicos y a menor precio.

En la figura 6 se representan gráficamente los valores medios de los potenciales de picadura frente a los de PREN, para cada aleación dúplex baja en níquel preparada durante la experimentación. En esta figura puede comprobarse como al aumentar el valor de PREN, aumenta el potencial de picadura, encontrándose que existe una relación de tipo exponencial entre ambas variables.

En la figura 7 se representan los potenciales de picadura de todos los materiales dúplex bajos en níquel y clásicos ensayados, frente a los valores

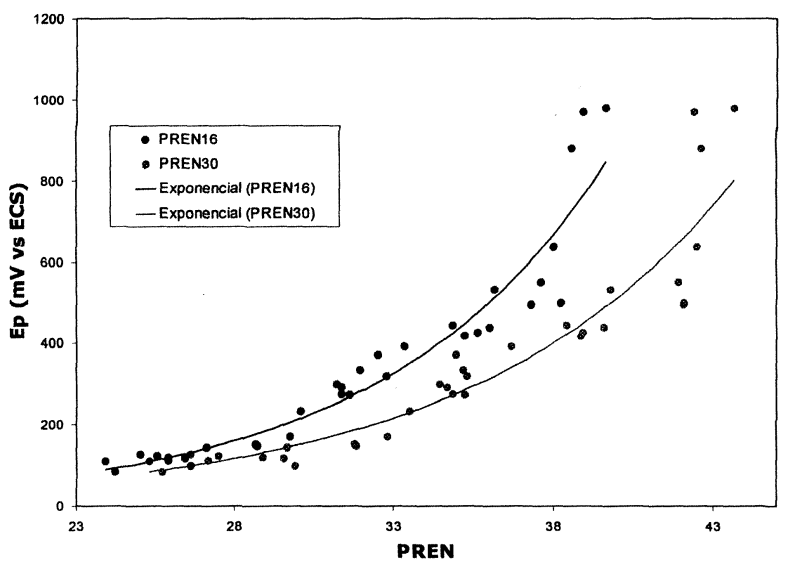

Figura 6. Representación gráfica de los valores obtenidos de potenciales de picadura (Ep) para las aleaciones dúplex bajas en níquel frente a PREN.

Figure 6. Ep obtained values from the duplex alloy with a low conte" in Ni Vs. PREN. 


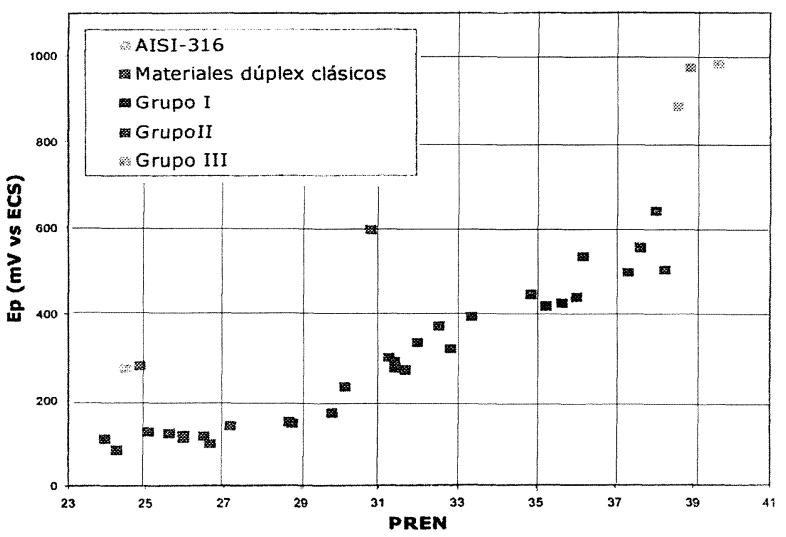

Figura 7. Valores de Ep de las aleaciones dúplex clásicas y bajas en níquel, frente a los valores de PREN.

Figure 7. Ep values from the classic and low content $\mathrm{Ni}$ duplex alloys Vs. PREN values.

calculados de PREN, para cada uno de ellos. Para los materiales dúplex bajos en níquel, a medida que aumenta el PREN, los valores de Ep son más altos. La tendencia seguida se ajusta a un modelo matemático exponencial. Para estos materiales se pueden distinguir tres grupos diferenciados en comportamiento, los cuales están señalados en la figura 7 .

Los materiales del grupo I cubren un rango de PREN comprendido entre los valores de 23,93 y 30,10 . Este grupo se caracteriza porque no hay un aumento significativo del valor de potencial de picadura a medida que los materiales están más aleados y, por tanto, tienen valores de PREN superiores. Todos los materiales incluidos en este grupo tienen contenidos de Mo $\leq 1 \%$. De esta forma, los resultados ponen de manifiesto que cuando el contenido de molibdeno se mantenga por debajo del $1 \%$, un aumento de los contenidos de cromo o nitrógeno, que se reflejaría en un incremento del valor de PREN, no se manifiesta en un aumento significativo del potencial de picadura en las condiciones experimentales consideradas $\left(50^{\circ} \mathrm{C}\right.$ y $50 \mathrm{~g} / \mathrm{l} \mathrm{de} \mathrm{NaCl}$ ). Estos resultados resaltan, una vez más, la importancia del contenido de molibdeno sobre el comportamiento frente a la corrosión por picaduras de estas nuevas aleaciones.

El grupo II se caracteriza porque, para una variación semejante del PREN a la comentada anteriormente, se produce una variación significativa en el valor del potencial de picadura. En este grupo, el contenido de molibdeno se sitúa entre el 2 y el $3 \%$. En tal caso, un aumento en el contenido de cromo y nitrógeno en la aleación, sí se traduce en una mejora de su comportamiento frente a la corrosión por picaduras, de forma que, un aumento en una unidad de PREN se traduce en un incremento de, aproximadamente, $50 \mathrm{mV}$ en el potencial de picadura.

Por último, en el grupo III se incluyen materiales con contenidos en molibdeno entre el 3 y $4 \%$. Este tipo de materiales se caracteriza porque, un aumento de una unidad en el valor del PREN se traduce en un incremento del potencial de picadura superior a $90 \mathrm{mV}$.

En definitiva, parece existir una relación entre el PREN y los potenciales de picadura de los dúplex bajos en níquel. El empleo de ecuaciones del tipo de la (4) permitiría diseñar dúplex bajos en níquel con resistencias a la corrosión por picaduras equivalente a las de los dúplex clásicos. Así, por ejemplo, el dúplex clásico 2304 tiene un potencial de picadura de $282 \mathrm{mV}$ y un PREN de 24,82 . Si se desea preparar un dúplex con bajo contenido en níquel, con resistencia a la corrosión por picadura equivalente a la de este material, se aplicaría la ecuación (4) para determinar qué valor de PREN sería necesario que tuviera el nuevo acero dúplex, bajo en níquel, para igualar las prestaciones del dúplex clásico. Es necesario tener en cuenta que, para que el acero diseñado tenga las óptimas propiedades mecánicas y de resistencia a la corrosión por picadura, debe tener una composición química equilibrada y una relación de fases adecuada.

\subsection{Microestructura de los materiales duplex bajos en níquel}

La figura 8 muestra la estructura de solidificación de estos nuevos aceros inoxidables dúplex con bajos contenidos en níquel. Esta estructura se

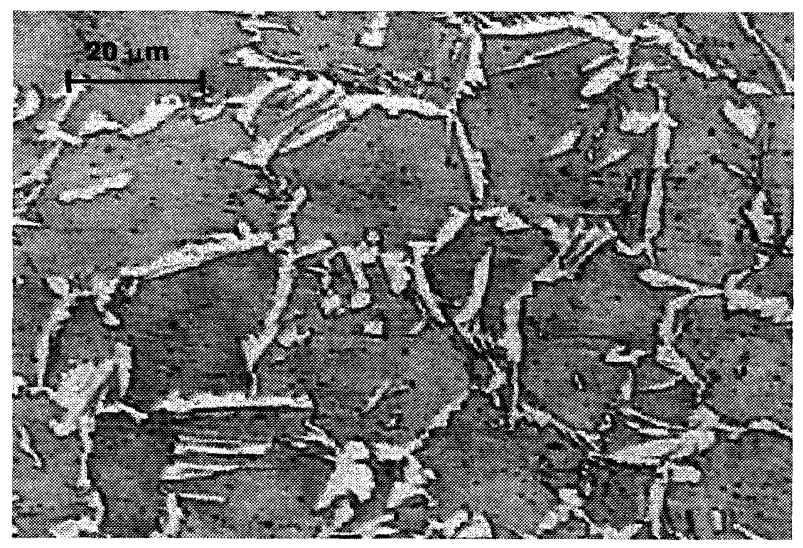

Figura 8. Microestructura de solidificación característica de los materiales dúplex de bajo contenido en níquel.

Figure 8. Solidification microestructure of a duplex material with low content $\mathrm{Ni}$. 
caracteriza como es habitual por una matriz ferrítica (color oscuro) donde se distribuyen las agujas de austenita (color claro).

$\mathrm{Al}$ someter las aleaciones dúplex a un tratamiento térmico de homogeneización de $1.100^{\circ} \mathrm{C}$, durante $30 \mathrm{~min}$, se observa una disminución del contenido de ferrita. Al mismo tiempo, las agujas de austenita se redondean, como se puede observar en la figura 9.

En la figura 10 se recogen las imágenes obtenidas al revelar la estructura de una aleación dúplex de bajo níquel, tras un ensayo potenciodinámico.

La imagen de la figura 11 muestra cómo las picaduras encontradas en la superficie de ensayo se en-

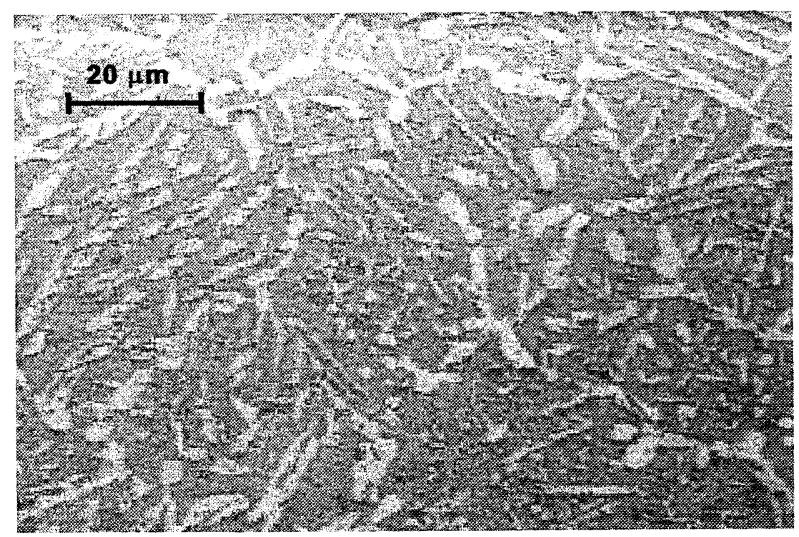

Figura 9. Microestructura característica de los materiales dúplex de bajo contenido en níquel después del tratamiento térmico de homogeinización $\left(1.100^{\circ} \mathrm{C}, 30 \mathrm{~min}\right)$.

Figure 9. Solidification microstructure of a Duplex material with low content $\mathrm{Ni}$ after homogeneitation heat treatment $\left(1100^{\circ} \mathrm{C}, 30 \mathrm{~min}\right)$.

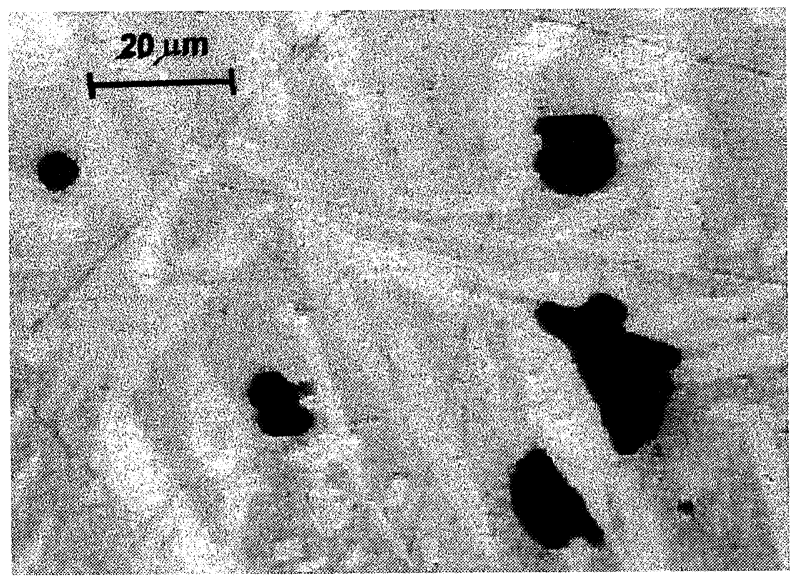

Figura 10. Imagen del dúplex de bajo níquel adquirida en un microscopio óptico después de un ensayo potenciodinámico.

Figure 10. Duplex material with low content $\mathrm{Ni}$ image obtained from an optic microscopy after a potenciodinamic test.

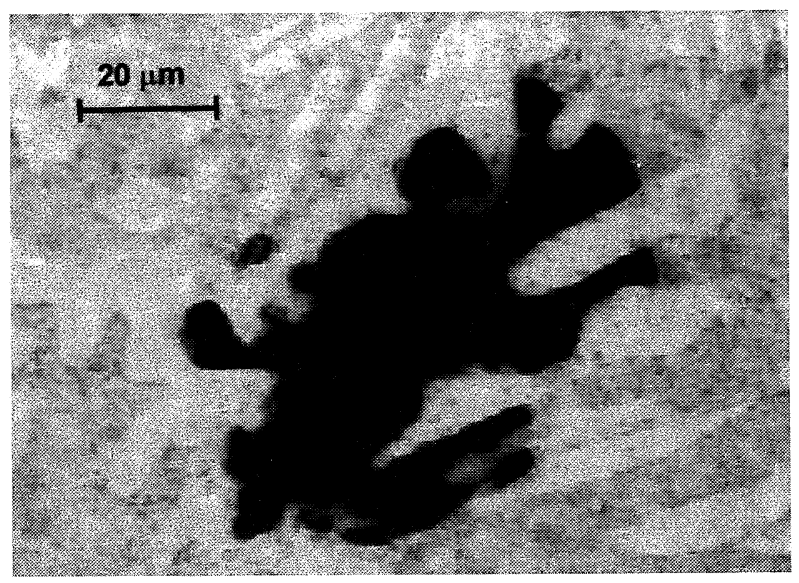

Figura 11. Imagen del dúplex de bajo níquel mostrando la disolución selectiva de ferrita.

Figure 11. Duplex material with low content $\mathrm{Ni}$ image, showing the phase a dissolution.

cuentran en la fase ferrita, comprobándose que el inicio de las picaduras se produce en esta fase. Las picaduras, al crecer, lo hacen disolviendo sólo ferrita e, incluso, se puede observar que bordean los granos de austenita, quedando estos últimos intactos.

En otras aleaciones ensayadas, con pequeñas diferencias en la composición que se discutirán más adelante, se observa (Fig. 12) que las picaduras de menor tamaño se encuentran en granos de austenita, de lo que se puede deducir que el ataque comienza en esta fase. Se observa, también, que las picaduras de mayor tamaño abarcan las dos fases, por lo que parece no hay disolución selectiva de fase austenita, que sufre el ataque inicial.

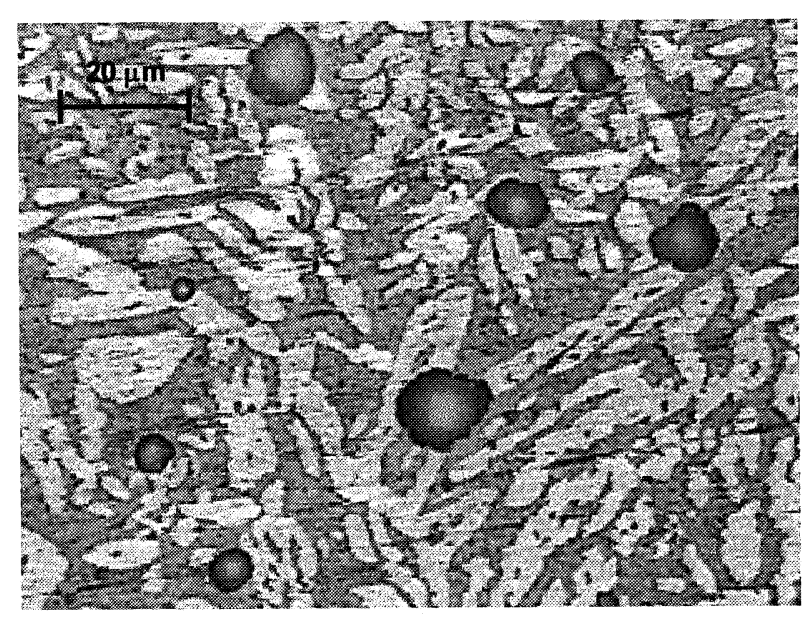

Figura 12. Imagen de un dúplex de bajo níquel con bajo valor de PREN de su fase g después de un ensayo.

Figure 12. Image of a duplexmaterialwith low content $\mathrm{Ni}$ and phase a PREN value, after a test.

Rev. Metal. Madrid 41 (2005) 148-158

http://revistademetalurgia.revistas.csic.es 
La figura 13 muestra la imagen de electrones secundarios, obtenida por Microscopía Electrónica de Barrido, de una picadura nucleada en la fase ferrita.

Se han analizado, por EDS, los cristales que quedan sin disolver en el interior de la picadura, verificándose que pertenecen a granos de austenita. Esta aleación tiene una microestructura homogénea formada por un $59,69 \%$ de fase ferrita y un $30,35 \%$ de austenita. El hecho de encontrar, sólo, cristales de austenita en el interior de la picadura, parece corroborar que se ha producido una disolución selectiva de fase ferrita.

Por otra parte, en la figura 14 , se muestran las imágenes obtenidas, tras la observación en el mi-

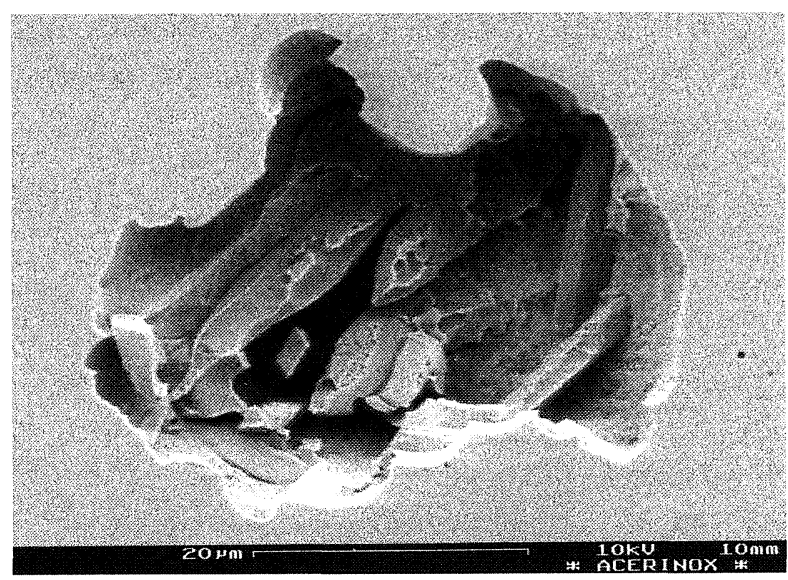

Figura 13. Imagen de electrones secundarios de una picadura de una aleación dúplex de bajo níquel con $\mathrm{PREN}_{a}<$ PREN.

Figure 13. Secondary electron image showing a pitt in a duplex material with low content $\mathrm{Ni}$ (PREN $\mathrm{P}_{a}<$ PREN).

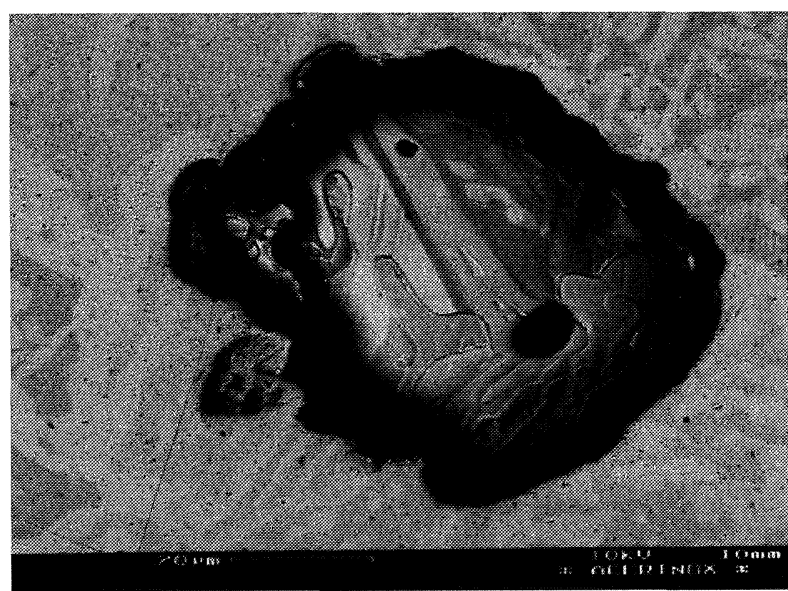

Figura 14. Imagen de electrones retrodispersados de una picadura de una aleación dúplex de bajo níquel con PREN $_{a}$ $>$ PREN $_{g}$

Figure 14. Backscattered electron image showing a pitt in a duplex material with low content $\mathrm{Ni}\left(\right.$ PREN $_{a}<$ PREN $\left._{g}\right)$

Rev. Metal. Madrid 41 (2005) 148-158 croscopio electrónico, de la superficie ensayada de una aleación en la que, por microscopía óptica, se había observado nucleación de picaduras en la austenita.

La imagen permite distinguir granos de ferrita (color oscuro) y de austenita (color claro) en el interior de la picadura. Este hecho, indicaría que no hay disolución selectiva de la austenita.

De acuerdo con lo comentado y en concordancia con otros autores ${ }^{[21 \text { y } 34]}$, el comportamiento a la corrosión de los aceros inoxidables dúplex, está determinado por la fase más débil. Por tanto, para estimar la relativa resistencia a la corrosión por picaduras de los aceros dúplex, debe de ser más apropiado usar el PREN de la fase más débil. Se ha comprobado experimentalmente que, efectivamente, la picadura nuclea en la fase con menor valor de PREN.

En relación con esto, cabe decir que el nitrógeno juega un papel muy importante en la definición de la fase más débil y, por tanto, es determinante en el comportamiento a la corrosión por picaduras del acero dúplex. En general, los materiales dúplex con bajos contenidos de nitrógeno tienen la fase austenita menos resistente que la ferrita, en la cual hay una mayor concentración de los elementos cromo y

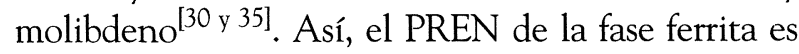
superior al de la austenita. Sin embargo, si se aumenta el contenido de nitrógeno, este se concentra, preferentemente, en la austenita, ya que su solubilidad en la ferrita está limitada ${ }^{[19}$ y 21-24]. De esta forma, el PREN de la fase austenita se puede igualar e, incluso, superar el de la ferrita. A la hora de diseñar un acero dúplex es importante tener en cuenta este hecho, ya que si las fases no tienen, aproximadamente, el mismo valor de PREN, la resistencia a la corrosión por picaduras de la aleación vendrá dada por el PREN de la fase más débil y, por tanto, será menor que la estimada por el valor de PREN calculado con la composición química global.

\section{REFERENCIAS}

[1] V. Matres, Tesis Doctoral, Facultad de CC. Químicas, Universidad Complutense de Madrid, 2000.

[2] R. Merello, Tesis Doctoral, Universidad de Cádiz, 2001.

[3] E. Alfonson y R. Quarfort, ACOM 1 (1992).

[4] M. Rener, U. Heubner, M.U. Rockel Y E. Wallis, Korros 37 (1986) 183-190.

[5] N. Suulata y M. Kurkela, Proc. Stainless Steels, Gothenburg, (1984) pp. 240-247.

[6] J.E. Truman, Proc U.K. Corrosion, Brighton, Oct. 26-28 (1987) pp. 111-129. 
[7] T. Kitada, Y. Kobayashi, M. TusujJ, T. Taira K. Ume y M. ITO, Nippon K Technical Report, Oversears 51 (1987) pp. 37-45.

[8] B. Vicentini, G. Rondelli Y A. Cigada, Wert. Korros 46(1995) 628-632.

[9] E. Otero, P. Karjalainen, M. Barteri y J. Botella, ECSC Research 7210Ma/95, 435, 983, 903, Confid. Report 1996.

[10] V. Matres y J. Botella, ECSC Research 7210MA/952, Confid. Report 1997.

[11] G. Wranglen, Sulfide Inclusion in Steel, ASM 6, Materials Metalworking Technology Series, 1975, pp.361-379.

[12] Z. SzKLARSKA-SmialowSKa. Sulfide Inclusion in Steel, ASM 6, Materials Metalworking Technology Series, 1975, pp.380 402 .

[13] M. Henthome. Sulfide Inclusion in Steel, ASM 6, Materials Metalworking Technology Series, 1995, pp. 445 458.

[14] R. Ajoudjov, C. Lemarie y G. Beranger, Corrosion Reviews 11(1993) 3.

[15] J. BAKer, E. Castle, Corrosion Science 34(1993) 667-682.

[16] J. Botella, V. Matres Y J. Almagro, Influencia de la relación (\% masa $\mathrm{Mn}$ ): (\% masa $\mathrm{S}$ ) en los aceros inoxidables $18 \mathrm{Cr} 8 \mathrm{Ni}$ y la composición química y estructural de los sulfuros formados en dichos aceros, Centro de Investigación de Acerinox, S.A.. Comunicación privada. 1998.

[17] J. Botella, V. Matres Y J. Almagro, "Relación entre las composiciones químicas de los sulfuros y la resistencia a la corrosión por picaduras de aceros 18Cr8Ni0,3S (AISI 303) con variables concentraciones de manganeso". Centro de Investigación de Acerinox, S.A.. Comunicación privada. 1998.

[18] A. Pardo, C. Merino, E. Otero, J. Botella, ECSC Research 7210Ma793y. Resistencia a la corrosión intergranular de acero inoxidable con níquel inferior al $2 \%$. Conf. Report 1991.

[19] Comunicación privada de los trabajos de la Tesis Doctoral de Inmaculada Moreno: Equilibrio de las fases a y $\mathrm{g}$ en aceros inoxidables dúplex austenoferríticos. Influencia de la composición química y de los coeficientes de reparto. Centro de Investigación y Desarrollo de Acerinox S.A., 2000.

[20] A. Van Bennekom, Stainless Steel World (1998) 54.

[21] L.F. Garfias-Mesías, J.M. Sykes, C.D.S. Tuck, Corros. Sci. 38 (1996) 1319.

[22] C.D.S. TUCK, J.M. SyKeS, L.F. GarfiAs-MeSÍAS, TWI paper 15, Glasgow (1994).

[23] S. Gutiérrez de Sáinz-Solabarría, J.M. San Juan NuÑEZ, Deformación metálica 226(1996).

[24] C.D.S. Tuck, L.F. Garfias-Mesías, J.M. Sykes, Duplex Stainless Steel'97 $5^{\text {th }}$ Conference, 1997. p. 683.

[25] A.J. SedRIKS, Corrosion of Stainless Steel. Second Edition, A. Wiley-Interscience Publication John Wiley \& Sons, Inc.

[26] R.A. Perren, P.J. Uggowiter, M.O. Speidel. Duplex Stainless Steel' $975^{\text {th }}$ Conference, 1997, p. 897.

[27] N. SRIDHAR, J. KolTS, Corrosion NACE 43(1987).

[28] M. Kemp, A. Van Bennekom, F.P.A. Robinson, Mater. Sci.Eng. A199 (1995) 183.

[29] Duplex Stainless Steels. Ed. By Robert N. Gunn (1997)

[30] S. Bernhardsson, Proc. of duplex Stainless Steel Conference, Beaune, France Vol. 1, 1991, p. 185.

[31] R.F.A. Jargelius-Pettersson, Corrosion 54 (1998) 162.

[32] H. HÄnninen, Materials Science Forum 318-320 (1999) 479.

[33] P.V. Scheers, J. Kerr, R. Paton, Proc. of $6^{\text {th }}$ Duplex Conference 2000, p. 31.

[34] H. Vannevik, J.O. Nilsson, J. Frodigh, P. Kangas, ISIJ Int. 36 (1996) 807.

[35] J. CHARLES, Welding in the World/Le Soudage dans le Monde 36 (1995) 43.

[36] M.I. Sarró, O. Alemán, D.A. Moreno, M. Roso, C. Ranninger, Rev. Metal. Madrid 40 (2004) 21-29.

[37] G. Fargas, J.M. Manero, M. Anglada, A. Mateo, Rev. Metal. Madrid 40 (2004) 219 - 223.

[38] A. Pardo, M.C. Merino, J. Botella, V. Matres, F. Viejo, M. Carboneras, R. Arrabal, Rev. Metal. Madrid 40 (2004) $335-340$. 